\title{
Paraneoplastic Pemphigus in a Patient with CARCinosarcoma of THE UTERUS
}

Georgia Giebel, MSIV and Gunjan Shah, MD

\section{Case Presentation}

The patient is a 68-year-old female with hypertension, chronic obstructive pulmonary disease, without medical follow-up for several years, who initially presented with skin blisters and oral lesions, which were biopsied by her dermatologist and found to be bullous pemphigoid. She was treated with a course of oral prednisone and had resolution of her skin and oral lesions. Three months later, she presented with a swollen leg and was diagnosed with a deep venous thrombosis, for which she was started on coumadin and had an inferior vena cava filter placed. She then had hemoptysis a few days later, after which the coumadin was held. A chest computed tomography (CT) done at that time found a right middle lobe consolidation consistent with aspiration pneumonia and a cavitary lesion in the left upper lobe of her lung, which was biopsied and found to be a necrotizing cavitary lung lesion. After being ruled out for tuberculosis, she had an outpatient positron emission tomography (PET) scan which was positive for a $2.7 \mathrm{~cm}$ left lower lobe cavitary lesion and a $9 \mathrm{~cm}$ pelvic mass with hypermetabolic activity, as well as small liver lesions. Concurrently, she complained of dysphagia and 30-lbs of weight loss over the prior six months, so the decision was made to undergo an outpatient esophagogastroduodenoscopy (EGD). The EGD could not be completed secondary to a stricture in the distal esophagus; however, pemphigus ulcers were seen in the mouth and esophagus. Given the patient's degree of malnourishment and dehydration, she was admitted to the outside hospital. On admission, she was found to have leukocytosis with a white blood cell count of $37,000 / \mathrm{L}$, hypercalcemia with a calcium of $13 \mathrm{mg} / \mathrm{dL}$, hypotension, and acute renal failure with a creatinine of $4.4 \mathrm{mg} / \mathrm{dL}$. Her parathyroid hormone-related protein $(\mathrm{PTHrP})$ was $105 \mathrm{pmol} / \mathrm{L}$ (normal $<1.3 \mathrm{pmol} / \mathrm{L}$ ), while her intact parathyroid hormone (PTH) was $<3 \mathrm{pg} / \mathrm{mL}(10-55$ $\mathrm{pg} / \mathrm{ml})$. A serum protein electrophoresis with immunoelectrophoreis was positive for an IgG lambda paraprotein. On bone marrow biopsy, there were $25 \%$ plasma cells consistent with multiple myeloma, though this diagnosis was questioned after transfer. For treatment of her hypercalcemia, she was given intravenous fluids and calcitonin with minimal improvement, but with resolution of her renal failure.

The esophageal biopsy showed squamous mucosa with necrotic ulcer and chronic inflammation with fibrinous exudate. The pelvic mass was also biopsied which was consistent with carcinosarcoma with massive necrosis. The pathology revealed myxoid high-grade spindle cell sarcoma with pleomorphosis and extensive mitosis and poorly differentiated adenocarcinoma with papillary serous differentiation, as well as some smooth muscle differentiation. During her hospitalization, the skin blisters reappeared and were biopsied. The biopsy was consistent with paraneo- plastic pemphigus. She was treated with three days of intravenous immunoglobulin (IVIg). She had an open jejunostomy tube placed for nutrition. At this point, she was transferred to Thomas Jefferson University for further management.

Initial vital signs after transfer were within normal limits aside from mild tachycardia. On physical exam, she was lethargic, but oriented to person and place. She had a normal physical exam other than a II/VI systolic ejection murmur, decreased breath sounds in her right lung base, and tenderness to palpation in the suprapubic region. She had no lymphadenopathy. A non-bleeding ulcer could be seen in her oral cavity, while her skin showed healing blisters (pictured below) without weeping. A pelvic exam was performed and a necrotic mass was found to be protruding into the cervix. Laboratory studies upon transfer included a white blood cell count of $32,800 / \mathrm{L}$, hemoglobin 8.5 g/dL, platelet count 362/L, CA-125 $1300 \mathrm{U} / \mathrm{mL}$ (normal 0-35 U/ $\mathrm{mL}$ ), creatinine $0.7 \mathrm{mg} / \mathrm{dL}$, and calcium $9.9 \mathrm{mg} / \mathrm{dL}$.

Dermatology was consulted and thought that her presentation was more consistent with paraneoplastic pemphigus given the combination of oral and skin lesions, rather than the previously diagnosed bullous pemphigoid. Hematology reviewed the initial bone marrow biopsy and concluded that it was more consistent with myoclonal gammopathy of unknown significance. Gynecology/Oncology stated that her prognosis was poor and that her best option was palliative radiation, as she was not a surgical candidate secondary to metastatic disease. Her hospital course was complicated by an additional episode of hypercalcemia causing mental status changes, and she was effectively treated with a dose of pamidronate. After palliative radiation, the patient was transferred to hospice care.

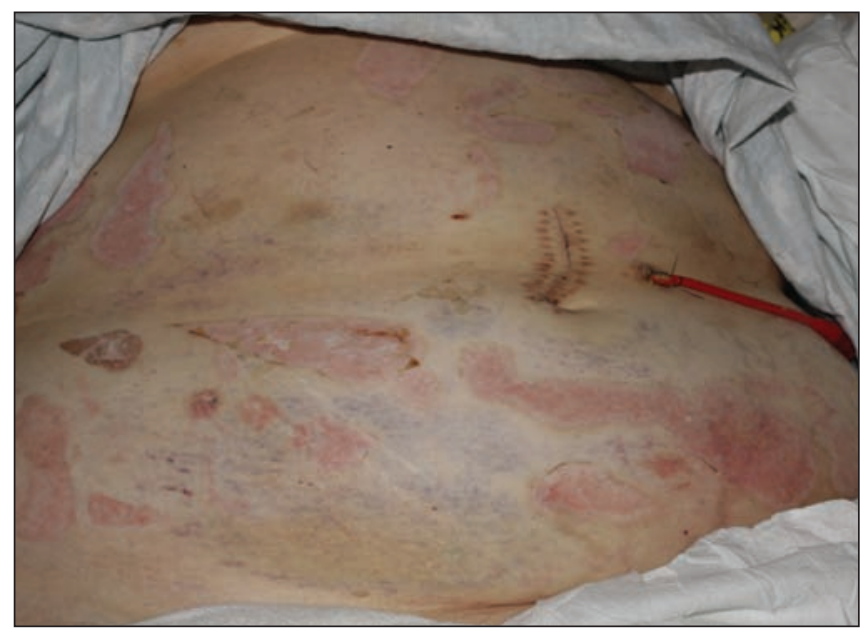

Figure 1. Healing skin blisters on abdomen 


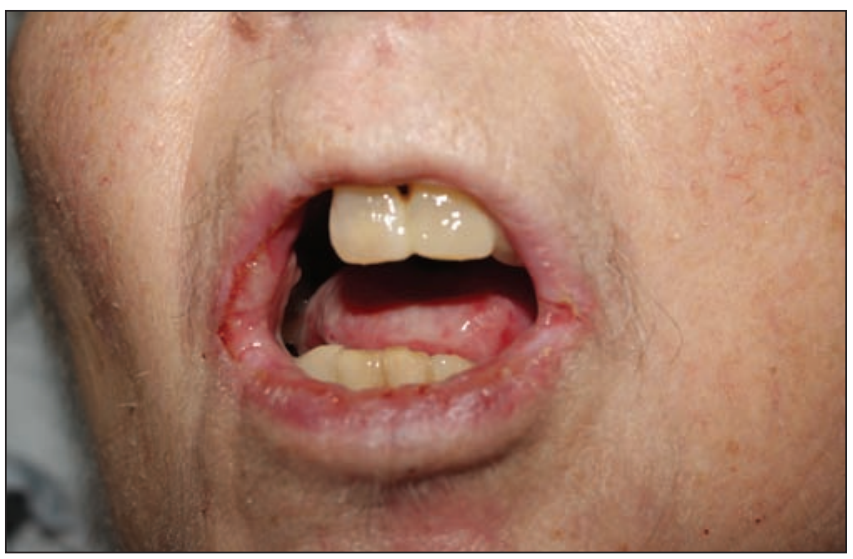

Figure 2: Oral ulcers
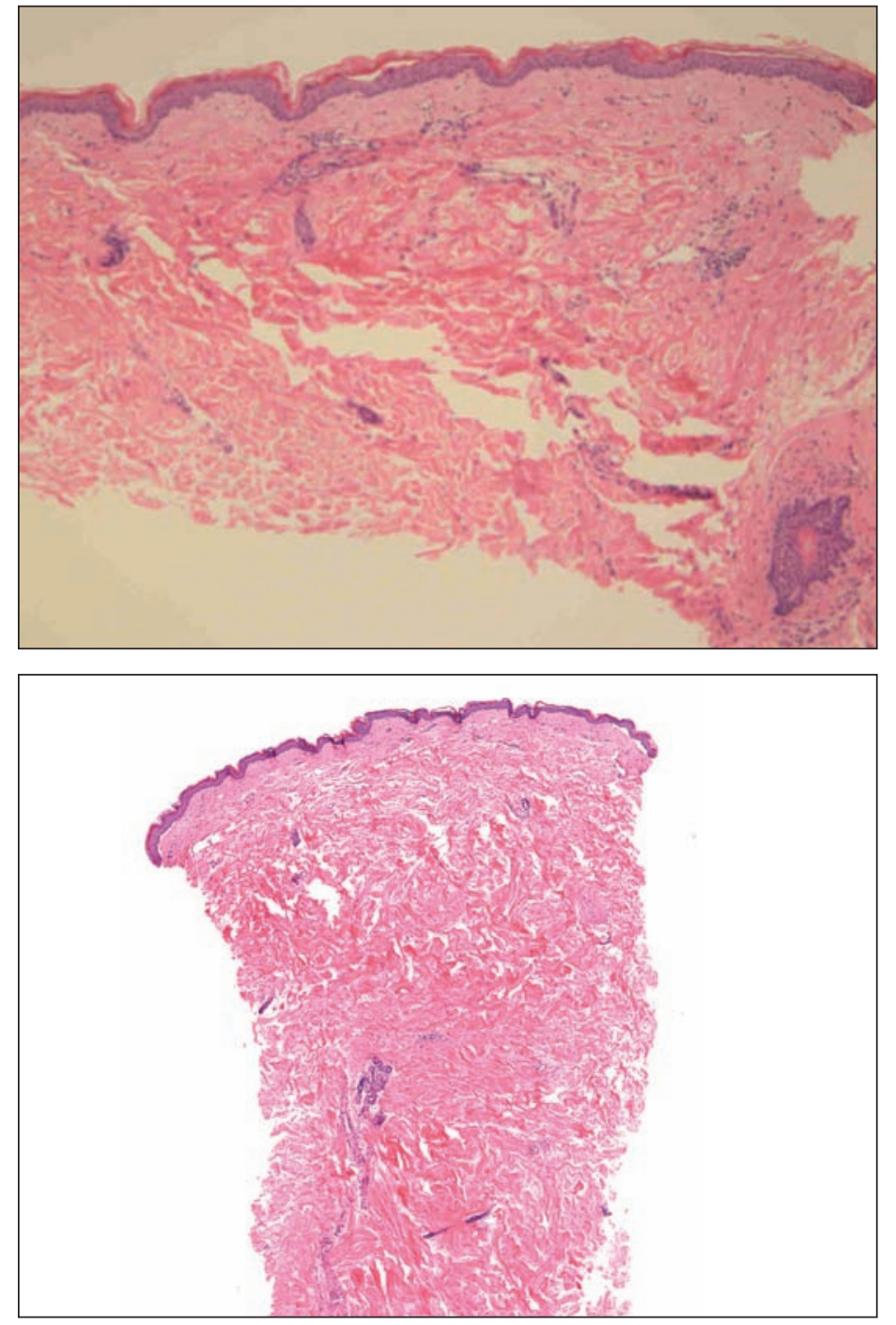

Figure 3: Skin biopsy pathology - H \& E Stain at 10x Magnification
A pauci-inflammatory subepidermal split can be seen at the lateral aspect of the punch biopsy. Our dermatopathologist believes that the split is not artifact, and represents abnormalities at the dermal-epidermal junction. This finding can also be seen in epidermolysis bullosa acquisita, bullous pemphigoid, other subepidermal bullous diseases, but taken in conjunction with the clinical history, it is consistent with paraneoplastic pemphigus.

\section{Discussion}

Paraneoplastic Pemphigus (PNP) is a rare, autoimmune blistering skin disorder, which occurs in the setting of a systemic neoplasm. The disease is characterized by polymorphous skin lesions, with a predilection for mucosal surfaces, along with evidence of IgG auto-antibodies against epidermal proteins. Paraneoplastic pemphigus was first described in 1990 by Anhalt et al. ${ }^{1}$, and since then there have been over 200 cases reported
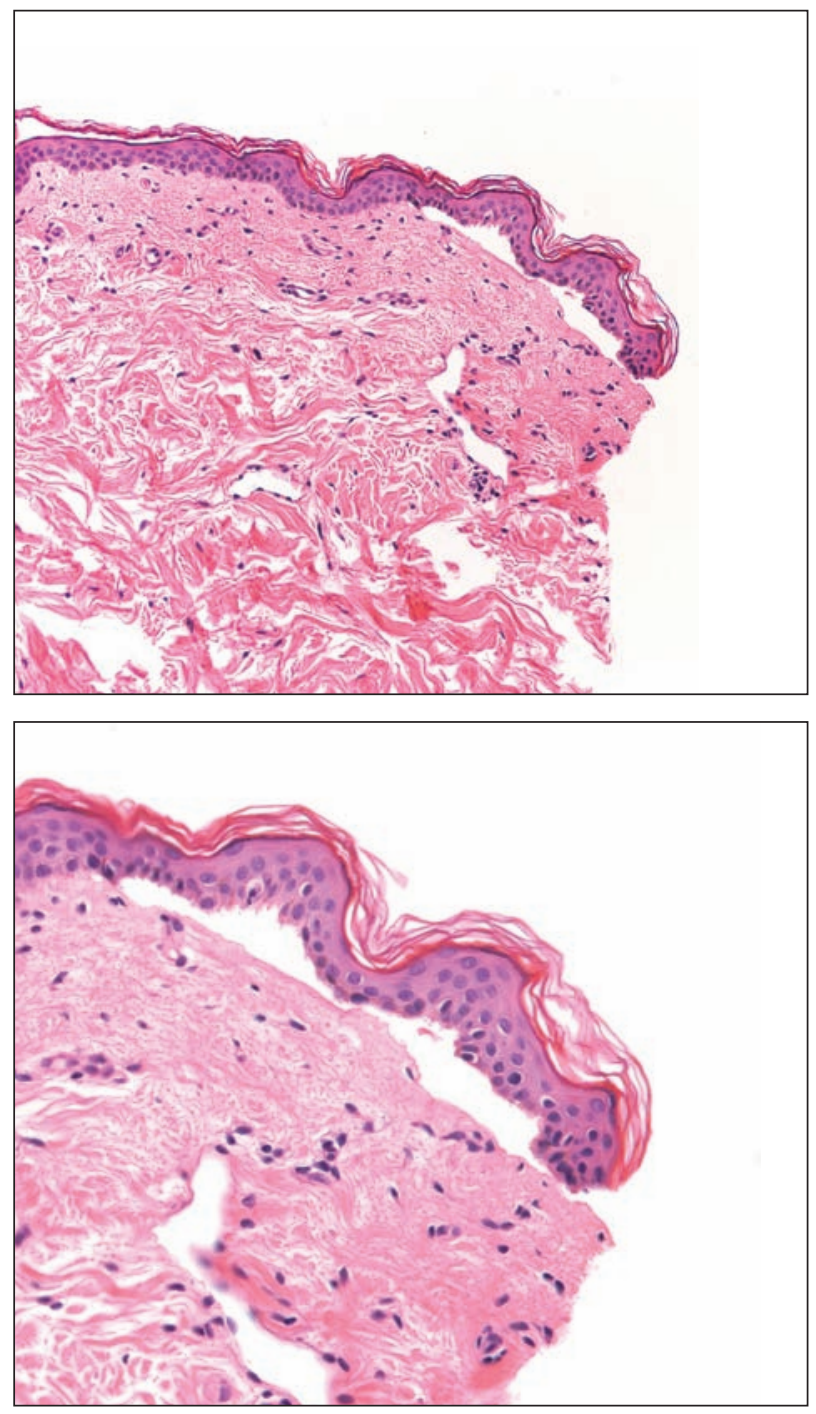
in the literature. Patients in all age groups have been affected by the disorder, including children and adolescents. ${ }^{2}$

Clinically, the presentation may be heterogeneous; however, mucosal lesions are inevitably present. Patients generally develop severe, painful stomatitis, and may have ulcerations and erosions of the oropharynx, larynx, esophagus, eyes, and genitalia. ${ }^{3}$ Cutaneous manifestations of the disease may vary in morphology. Skin lesions resembling erythema multiforme, graft-versus-host disease, lichen planus, as well as pemphigus and pemphigoid have been reported. ${ }^{3}$ Cutaneous findings generally appear after the onset of mucosal eruptions and may erupt in waves. Additionally, bronchiolitis obliterans may be a complication of PNP, often leading to respiratory failure and death. ${ }^{4,5}$ Bronchial inflammation and destruction with subsequent fibrosis appears to be caused by deposition of IgG autoantibodies on bronchial epithelial cell surfaces and basement membranes. ${ }^{6}$ Onset of dyspnea and dry cough in patients with paraneoplastic pemphigus should warrant prompt attention and therapy in order to avoid rapidly progressive respiratory complications.

PNPisassociated withavarietyof neoplasms. Lymphoproliferative disorders predominate, with non-Hodgkin's lymphoma, chronic lymphocytic leukemia, Castelman's tumor, and thymoma being the most common. ${ }^{7}$ Of note, PNP with monoclonal gammopathy has been reported as well. ${ }^{8}$ Non-hematologic neoplasms associated with PNP are liposarcoma, leiomyosarcoma, adenocarcinoma of the breast, colon, pancreas and other organs, basal cell carcinoma, and malignant melanoma. ${ }^{9}$

The histopathology in PNP varies according to the morphologic characteristics of the cutaneous lesions. Horn \& Anhalt ${ }^{10}$ studied the histology of 16 biopsy specimens from six patients with PNP, and determined that there are a number of major features characteristic of the disorder. Acantholysis, or the loss of intercellular connections between epidermal cells, was commonly noted in the suprabasal region. Dyskeratotic keratinocytes, or the abnormal keratinization of skin cells characterized by eosinophilic, shrunken keratinocytes, was commonly seen at all epidermal levels. Suprabasal clefts or blisters reminiscent of those seen in pemphigus vulgaris, basal vascularization, and inflammatory cell exocytosis into the epidermis were other major features noted. ${ }^{10}$ The authors determined that both acantholysis and dyskeratotic keratinocytes at all levels of the epidermis indicate the presence of PNP.

A number of autoantibodies have been isolated in patients with PNP. Antibodies to the plakin family of proteins, which are structural proteins in hemidesmosomal and desmosomal plaques, have been reported. ${ }^{11}$ Anti-envoplakin and anti-periplakin have been noted to be the most specific autoantibodies. ${ }^{8}$ Anti-desmogelin-3 and anti-desmoglein- 1 are also commonly isolated proteins. Autoantibodies may be detected using immunoprecipitation of antigen complexes, Western blot, and ELISA. ${ }^{11}$ It is likely that the diversity of autoantibodies accounts for the wide clinical spectrum of PNP.
Immunofluorescence, both direct and indirect, is an important component of the diagnosis of PNP. On direct immunofluorescence, deposits of IgG and complement in the epithelium are distributed both intercellularly and at the basement membrane zone. ${ }^{10,11}$ Indirect immunofluorescence may be used to detect antibodies in sera, utilizing monkey esophagus or rat bladder epithelium ${ }^{12}$. Using rat bladder as a substrate is more specific for PNP, as autoantibodies found in pemphigus vulgaris do not bind to transitional epithelia. However, indirect immunofluorescence may be negative in as many as one fourth of patients, and in this case, immunoprecipitation should be used to aid in the diagnosis. ${ }^{13}$

There have been a number of hypotheses regarding the pathogenesis of PNP. Xuejun and Bingxin ${ }^{14}$ isolated B-lymphocyte clones from a Castleman's tumor, which specifically reacted to epidermal proteins, indicating that the associated tumors may produce auto-antibodies. Other authors have postulated that paraneoplastic skin eruptions promote exposure of self-antigens and subsequent development of auto-antibodies. ${ }^{15}$ Epitope spreading may then occur, where tissue damage exposes additional self-antigens, thereby diversifying the auto-antibodies to recognize additional proteins. ${ }^{16}$

Criteria for diagnosis were devised by Anhalt and colleagues in their original article. ${ }^{1}$ Those criteria have been revised into major and minor features. Major features include polymorphic mucocutaneous eruption, concomitant internal neoplasia, and characteristic serum immunoprecipitation findings. Minor criteria include evidence of acantholysis on histology, direct immunofluorescence staining of intercellular and basement membrane staining, and indirect immunofluorescence staining of rat bladder epithelium. Patients meeting all three major criteria and at least two minor criteria should be considered to have PNP. ${ }^{17}$

Treatment of PNP includes early detection and resection of the associated tumor, especially in the case of Castleman's disease. Intravenous immunoglobulin (IVIg) should be administered before, during, and after the operation. ${ }^{14}$ Immunosuppression is an important component of treatment of PNP, and high-dose corticosteroids are first line. ${ }^{17}$ Cyclosporine, azathioprine ${ }^{11}$, mycophenolate mofetil, ${ }^{18}$ and other steroid-sparing agents may be used as adjunct therapy. If these agents fail to control the disease, alternative agents such as plasmapheresis, $\operatorname{IVIg}^{14}$, and rituximab ${ }^{19,20}$ have been used with mixed results. Unfortunately, response to treatment is generally poor, and PNP frequently results in premature death compared to the average life expectancy associated with the underlying malignancy., ${ }^{3,15}$ The prognosis is generally more favorable in patients with benign neoplasms, such as Castleman's syndrome or thymoma ${ }^{3}$.

In summary, paraneoplastic pemphigus is a rare, polymorphous blistering disease with a predilection for the mucosal surfaces in the setting of an associated neoplasm. The disease should be suspected if histology demonstrates acantholysis and 
dyskeratotic kerotinocytes, and if characteristic immunofluorescence is noted. Prompt diagnosis and treatment, along with search for underlying neoplasm, is appropriate as progression of the disease can result in death.

\section{References}

1. Anhalt GJ, Kim SC, Stanley JR, et al. Paraneoplastic pemphigus. An autoimmune mucocutaneous disease associated with neoplasia. N Engl J Med 1990; 323: 1729-1735.

2. Mimouni D, Anhalt GJ, Lazarova Z, et al. Paraneoplastic pemphigus in children and adolescents. Br J Dermatol 2002; 147: 725-732.

3. Nguyen VT, Ndoye A, Bassler KD, et al. Classification, clinical manifestations, and immunopathological mechanisms of the epithelial variant of paraneoplastic autoimmune multiorgan syndrome: a reappraisal of paraneoplastic pemphigus. Arch Dermatol 2001; 137: 193-206.

4. Takahashi M, Shimatsu Y, Kazama T, et al. Paraneoplastic pemphigus associated with bronchiolitis obliterans. Chest 2000; 117(2): 603-607.

5. Mar WA, Glaesser R, Struble K, et al. Paraneoplastic pemphigus with bronchiolitis obliterans in a child. Pediatr Dermatol 2003; 20: 238-242.

6. Nousari HC, Deterding R, Wojtczack H, et al. The mechanism of respiratory failure in paraneoplastic pemphigus. N Engl J Med 1999; 340: 1406-1410.

7. Sehgal VN, Srivastava G. Paraneoplastic pemphigus/paraneoplastic autoimmune multiorgan syndrome. Int J Dermatol. 2009; 48(2): 162-169.

8. Joly P, Richard C, Gilbert D, Courville P, et al. Sensitivity and specificity of clinical, histologic, and immunologic features in the diagnosis of paraneoplastic pemphigus. J Am Acad Dermatol 2000; 43:619-626.
9. Kaplan I, Hodak E, Ackerman L, et al. Neoplasm associated with paraneoplastic pemphigus: a review with emphasis on non-hematologic malignancy and oral mucosal manifestations. Oral Oncology 2004; 40: 553-562.

10. Horn TD, Anhalt GJ. Histologic features of paraneoplastic pemphigus. Arch Dermatol 1992; 128: 1091-1095.

11. Zimmerman J, Bahmer F, Rose C, et al. Clinical and immunopathological spectrum of paraneoplastic pemphigus. J Dtsch Dermatol Ges 2010; 8(8): 598-606.

12. Helou J, Allbritton J, Anhalt G. Accuracy of indirect immunofluorescence in the diagnosis of paraneoplastic pemphigus. J Am Acad Dermatol 1995; 32: 441-447.

13. Liu AY, Valenzuela R, Helm TN, et al. Indirect immunofluorescence on rat bladder transitional epithelium: A test with a high specificity for paraneoplastic pemphigus. J Am Acad Dermatol 1993; 28: 696-699.

14. Zhu X, Zhang B. Paraneoplastic Pemphigus. J Derm 2007; 34: 503-511

15. Bowen GM, Peters NT, Fivenson DP, et al. Lichenoid dermatitis in paraneoplastic pemphigus. Arch Dermatol 2000; 136: 652-656.

16. Chan LS. Epitope spreading in paraneoplastic pemphigus: autoimmune induction in antibody-mediated blistering skin diseases. Arch Dermatol 2000; 136; 663-664.

17. Camisa C, Helm TN. Paraneoplastic Pemphigus is a distinct neoplasia-induced autoimmune disease. Arch Dermatol 1993; 129: 883-886.

18. Williams JV, Marks JG Jr, Billingsley EM. Use of mycophenolate mofetil in the treatment of paraneoplastic pemphigus. Br J Dermatol 2000; 142: 506-508.

19. Schadlow MB, Anhalt GJ, Sinha A. Using rituximab (anti-CD20 antibody) in a patient with paraneoplastic pemphigus. J Drugs Dermatol 2003; 2: 564-567.

20. Bamadas M, Roe E, Brunet S, et al. Therapy of paraneoplastic pemphigus with Rituximab: a case report and review of the literature. J Eur Acad Dermatol Venereol 2006; 20(1): 69-74.

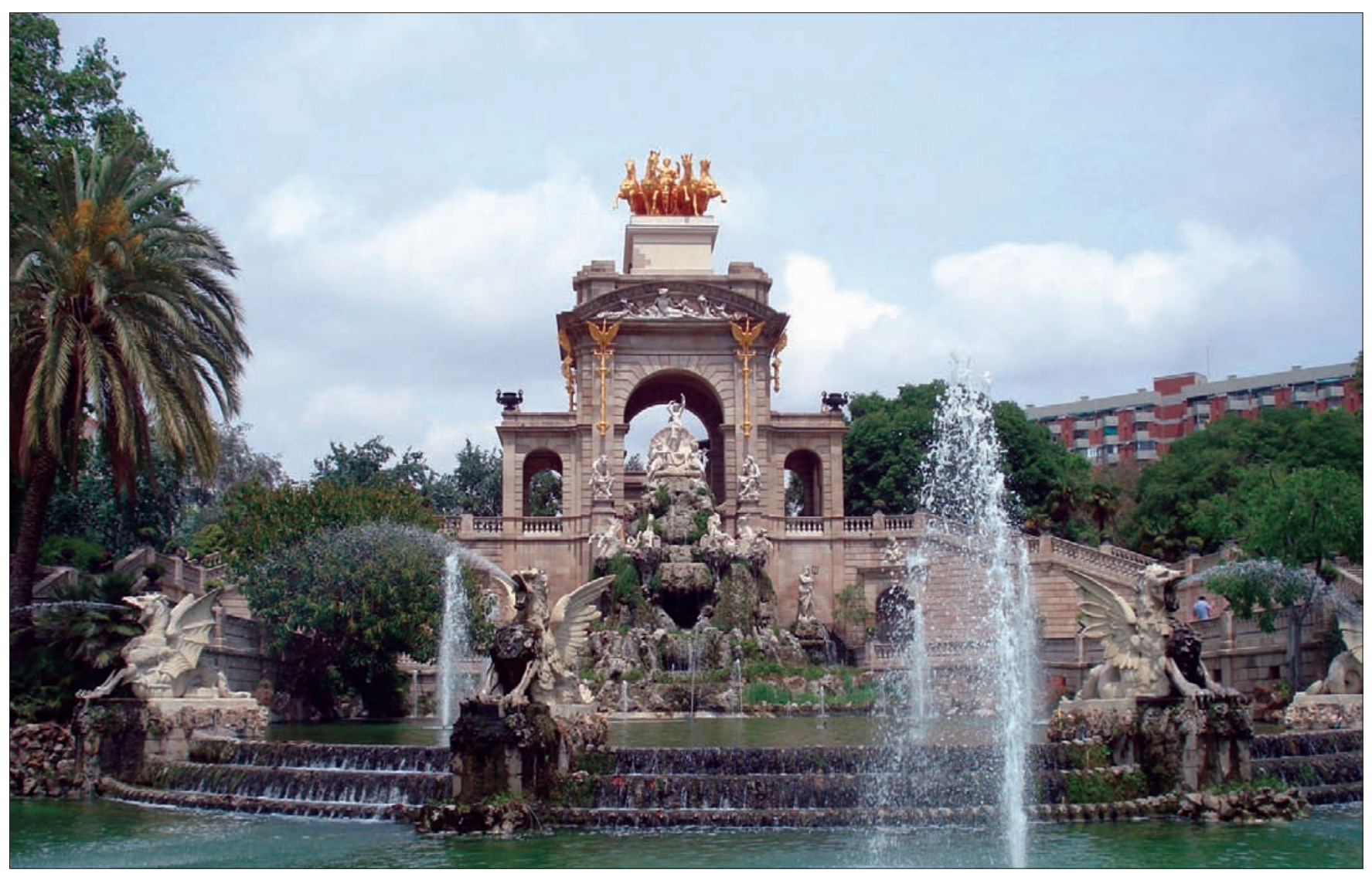

"Parc de la Ciutadella, Cascada Fountain", photograph by Paurush Shah, MD 